\title{
A Framework for Health Behavior Change using Companionable Robots
}

\author{
Bandita Sarma \\ University of North Texas \\ banditasarma@my . unt . edu
}

\author{
Amitava Das \\ University of North Texas \\ Amitava.Das@unt.edu
}

\author{
Rodney D. Nielsen \\ University of North Texas \\ Rodney.Nielsen@unt.edu
}

\begin{abstract}
In this paper, we describe a dialogue system framework for a companionable robot, which aims to guide patients towards health behavior changes via natural language analysis and generation. The framework involves three broad stages, rapport building and health topic identification, assess patient's opinion of change, and designing plan and closing session. The framework uses concepts from psychology, computational linguistics, and machine learning and builds on them. One of the goals of the framework is to ensure that the Companionbot builds and maintains rapport with patients.
\end{abstract}

\section{Introduction}

Human beings engage in many activities or behaviors that can aggravate existing health problems or lead to new ones. Abandoning such behaviors with the help of motivational interviewing or counseling sessions is called health behavior change. Providing counseling sessions that result in behavior change is a difficult task even for expert practitioners, and hence poses a great challenge for automated dialogue systems. The process demands constant monitoring and an in-depth understanding of the patient ${ }^{1}$. A wrong move on the counselor's part could undo what might otherwise be a successful attempt to bring about the targeted health behavior change. This could require the counselor to return to the first stage of the process and regain the patient's trust.

In this paper, we describe a framework for a companionable robot, which can counsel its human companion and assist them in making healthier choices for a better life. In our previous work

\footnotetext{
${ }^{1}$ The terms patient and user are used interchangeably throughout the paper to refer to the human using the Companionbot.
}

(Nielsen et al., 2010), we described an overall architecture for a companion robot capable of proactive dialogue with patients. This paper focuses on a natural language processing framework for health behavior change dialogue. Bickmore and Sidner (2006) outline a plan-based framework, COLLAGEN, based on the transtheoretical model and motivational interviewing to generate health behavior change counseling dialogue for physical activity promotion. COLLAGEN conducts a session in four steps: greeting exchange, discussion of previous day's exercise, discussion of plans for next day, and finally, farewell exchange. In addition to having similar steps, our framework also discusses in detail the natural language processing modules that are involved in judging the user's mindset at each step and guiding him/her towards making a decision on changing health behavior. In their follow up work (Bickmore et al., 2009), they discuss several issues such as minimizing repetitiveness in the behavioral, linguistic and visual aspect of the agent, establishing a therapeutic alliance between the user and the agent for a successful dialogue, maintaining continuity over multiple sessions, and the challenge of open-ended question generation. In addition to these issues, there might be verbal resistance from the patient to the suggestions by the Companionbot.

Use of telemedicine is becoming a common practice in providing remote clinical health care. It involves the use of various technologies like telephone, Facsimile, e-mail, video meetings, etc. to provide medical services. However, telemedicine is not flexible and adaptive, or when it is, it requires a human in the loop. It might also require long wait times on the patient side to receive a response from a health expert. Using companionable robots to provide guidance for health behavior change can provide greater flexibility compared to standard telemedicine practices. Bajwa (2010) described a virtual medical expert system 
that leverages natural language processing techniques to provide medical help to user queries from a knowledge base using pattern matching. In case the query does not have a match in the knowledge base, it is directed to an expert. The present work is along similar lines in terms of providing medical advice but in case of an unknown health condition, the Companionbot provides information through Web search. In addition to this, our framework adds the capability of generating small talk, which will help the user overcome inhibitions that might arise in talking to a robot instead of a human. The medical advice provided by the Companionbot will be in the form of suggestions rather than instructions. This is intended to make users reflect on their own choices comfortably instead of receiving instructions from the Companionbot's advice. The Nursebot project (Roy et al., 2000) discussed five different functions to assist the elderly through personal robots. One of the functions is to provide virtual telemedicine based facilities. Another robot called Paro (Kidd et al., 2006) was developed to cater to the social needs of elderly in nursing homes and was capable of generating a small set of vocal utterances in addition to limited voice recognition and body movement. Our framework, when implemented successfully, will be capable of engaging the user in a complete conversation, both casual and therapeutic.

\section{Framework}

The proposed dialogue system framework consists of three broad stages. The first stage aims to build rapport with the patient and identify the health topic to be discussed. The second stage involves identifying the issues and challenges the patient perceives associated with enacting relevant health behavior changes and motivating the patient to make the most appropriate change(s). The final stage summarizes the overall plans and goals, and encourages the patient to follow through. The entire process from building rapport with the patient through motivating changes in health-related behavior may span several sessions, and of course, is likely to be repeated for other behaviors.

\subsection{Build rapport \& identify health topic}

In order to initiate a counseling session it is essential to build and maintain rapport with the patient. This helps the patient feel more comfortable with the situation, which facilitates more open commu- nication. Reasonable rapport needs to be established in the initial stages when the Companionbot is first introduced to the patient. However, since the Companionbot is meant to be present constantly with its human companion, rapport building and maintenance is expected to be an ongoing process. Throughout both the casual and health behavior change dialogue, the Companionbot will identify the patient's interpersonal relations, health conditions and beliefs, likes and dislikes, habits, hobbies, and routines. These will be stored in a user model, which the language generation engine will exploit to engage the user in dialogue that is guided by, and infused with, personal context. A language understanding component will constantly assess the user's engagement level in the conversation. If the user seems to be disinterested at any point, a Typical Day strategy (Mason and Butler, 2010) is used to deal with the situation where the Companionbot will ask the user what a typical day for them is like.

When the system has achieved an adequate level of rapport, the next step is to identify a health topic of concern to the patient, so that there can be a focused discussion geared towards health behavior change. The present project will start with a small list of conditions and the behaviors that, when altered, can bring about an improvement in the condition. For example, heart disease includes diet and exercise, among others, as the associated behaviors. These conditions will be identified primarily using named-entity recognition and keyword spotting. If the Companionbot identifies heart disease as the topic, then the discussion could focus on food habits or exercise related issues.

\subsection{Assess patient's opinion of change}

Once a health concern is identified, the next step is to determine how important the patient thinks it is to change the associated behaviors and how confident they are about enacting those changes. This is an important stage because not all people have the same mindset regarding behavior change. Some might understand the importance of it but are not confident about achieving it while others might not consider it important at all. In order to understand the user's mindset, Mason and Butler (2010) suggest asking the user to assign cardinal values to quantify these opinions. The values may be on a scale of 0 to 10 , where 0 is the lowest and 


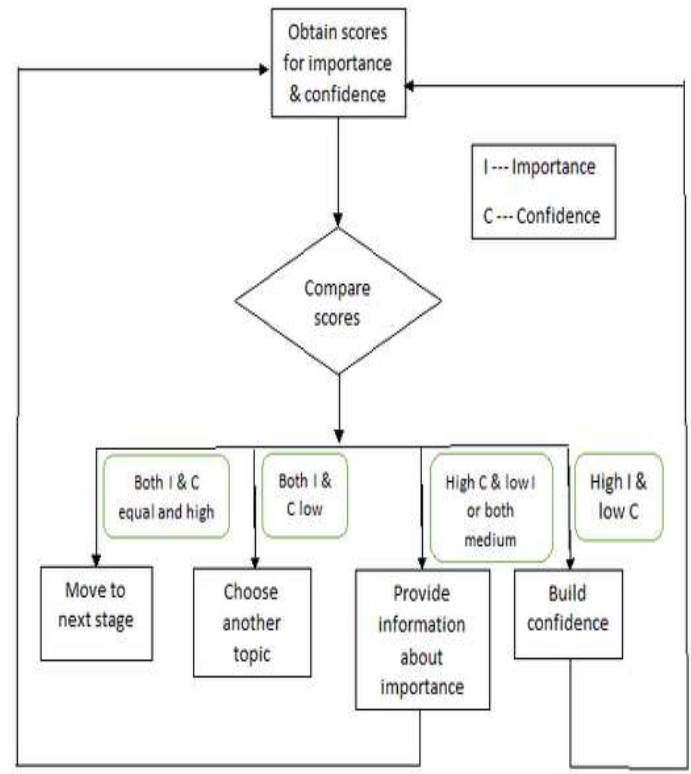

Figure 1: Block diagram for assessing patient's opinion of change

10 is the highest.

If there is a large difference between the user ratings of importance and confidence, the Companionbot will discuss the lower-ranked factor first (Mason and Butler, 2010). If the scores are approximately equal (e.g., the patient gives both importance and confidence a medium rating), then the Companionbot's dialogue will focus on helping the user understand the importance of the behavior change (Mason and Butler, 2010). Low values for both importance and confidence scores indicate that the user is not ready for these health behavior changes (Mason and Butler, 2010), and the Companionbot should move on to a different health topic or behavior. If both the scores are high, the Companionbot can move on to the next stage, summarizing the discussion and motivating the behavior changes. Figure 1 shows the block diagram representation for this module.

\subsection{Design plan \& close the session}

The Companionbot moves toward concluding the conversation by asking an open-ended question regarding how the user feels about the health behavior changes that they have been discussing. A user's attitude can be categorized into one of three categories, ready for change, not ready for change, or ambivalent. If the patient is ready for change, the Companionbot will provide suggestions on how to bring about the change in the be-

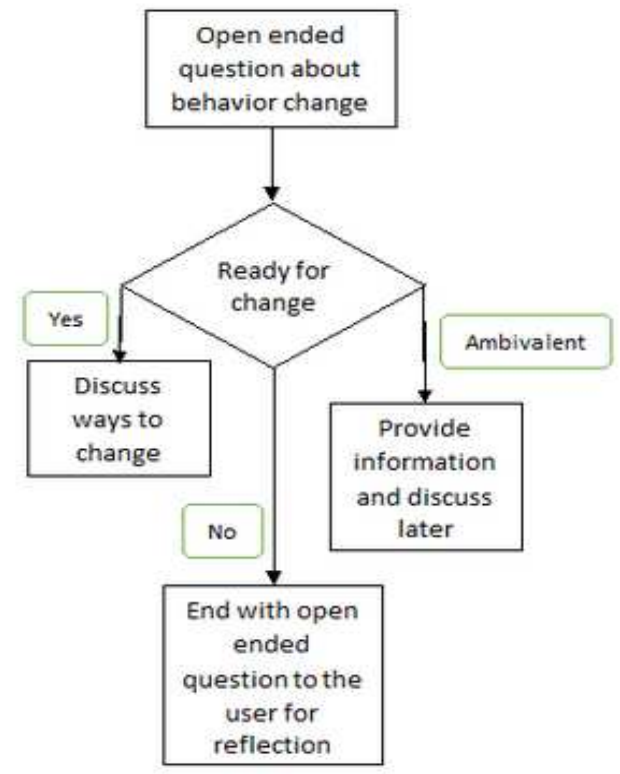

Figure 2: Block diagram for designing plan and closing the session

havior in previous step by leveraging knowledge from the user model and the conversation history. There may be patients who belong to the second category and are not ready for the health behavior change. We have already discussed ways on how to tackle such a situation in Subsection 2.2. If the patient is ambivalent about changing a behavior, the Companionbot will close by providing information to help the patient reflect on the pros and cons of the health behavior change until it is appropriate to bring it up again in a future session. A knowledge base will be maintained about the behaviors associated with common and critical health conditions. Information about a health condition, which is outside the domain of current knowledge base, will be retrieved using Web search. Figure 2 shows the block diagram representation of this stage.

If a session exceeds a pre-defined time, deemed to be the limit of most patients' ability to stay adequately focused on health behavior change, or if the system recognizes that the patient is losing their focus, the Companionbot will check-in with the patient, and if appropriate, will bring the session to a close following strategies that parallel those described in the preceding paragraph.

\section{Challenges}

Automatic generation of dialogue becomes a particularly challenging task when its purpose is to 
guide people through sensitive or personal issues like health behavior change. Some patients may not like to be told what is good or bad for them. In such a case, the patient might begin resisting suggestions for change (Mason and Butler, 2010). This places the entire counseling session in a precarious position and any wrong move on the Companionbot's part could push the patient to a higher level of resistance. To mitigate this scenario, the Companionbot will include patient resistance detection in the framework. If mild resistance is detected, the discourse is automatically directed towards bringing the user back on track. Whereas if there is high resistance, the Companionbot moves on to a different topic In case the user continues resisting then the Companionbot will close the session.

For successful implementation of therapeutic dialogue systems, it is essential to ensure that they do not sound monotonous. This is possible only if the responses are generated dynamically and hardcoding is limited. During rapport building and user modeling, questions will be generated by the Companionbot from various sources like the Internet, medical forms, information provided by physicians, family members, etc. At other times, responses will be constructed using both syntactic and semantic information from the user utterances.

Since multiple sessions might be held with the user to discuss a specific behavior, it is necessary to maintain continuity between the sessions (Bickmore et al., 2009). Bickmore and Sidner (2006) advocate dedicating a part of the dialogue to reviewing prior discussions, associated actions, and patient plans, as well as discussing what the patient has done since the last session to follow though on their plans. The Companionbot maintains a detailed user model including logs of the previous sessions, which will be used to review prior discussions, plans and actions and to guide ongoing motivational interviews.

Another challenge is choosing appropriate evaluation measures to determine the system's usefulness in bringing about the desired change in the patient. The efficacy of the system will be judged by monitoring the users behavior regularly. Any noticeable changes, such as weight gain or loss and increased or decreased smoking, will be tracked. How frequently a patient interacts with the Companionbot is an implicit qualitative measure of how much they appreciate it. We also plan to use questionnaires to elicit user ratings of the system for its acceptability and quality on a Lickert scale (Lickert, 1932).

\section{Conclusion}

In this paper we proposed a novel framework for automatic health behavior change counseling. Successful implementation of this framework would mean that the Companionbot could be used to guide patients towards bringing changes in their behavior for a healthier life. This can reduce the long wait period in conventional telemedicine practices from the time the patients contact the remote heatlh care provider to the instance they receive the instruction (Bajwa, 2010). Since the Companionbot will be capable of small talk aimed at connecting with the user on an emotional level, we hypothesize it will be perceived as being much more natural than existing conversational robots.

\section{References}

Cory D. Kidd, Will Taggart and Sherry Turkle. 2006. A Sociable Robot to Encourage Social Interaction among the Elderly. IEEE International Conference on Robotics and Automation, 3972-3976.

Imran S. Bajwa. 2010. Virtual Telemedicine Using Natural Language Processing. International Journal of Information Technology and Web Engineering, 5(1):43-55.

Nicholas Roy, Gregory Baltus, Dieter Fox, Francine Gemperle, Jennifer Goetz, Tad Hirsch, Dimitris Margaritis, Michael Montemerlo, Joelle Pineau, Jamieson Schulte and Sebastian Thrun. 2000. Towards Personal Service Robots for the Elderly. Workshop on Interactive Robots and Entertainment.

Pip Mason and Christopher C. Butler. 2010. Health Behavior Change. Elsevier Health Sciences.

Rensis Likert. 1932. A Technique for the Measurement of Attitudes. Archives of Psychology, 140:1-55.

Rodney D. Nielsen, Richard Voyles, Daniel Bolanos, Mohammad H. Mahoor, Wilson D. Pace, Katie A. Siek and Wayne H. Ward. 2010. A Platform for Human-Robot Dialog Systems Research. In Proceedings of AAAI Fall Symposium, Dialog with Robots, 161-162.

Timothy W. Bickmore and Candace L. Sidner. 2006. Towards Plan-based Health Behavior Change Counseling Systems. In proceedings of AAAI Spring Symposium on Argumentation for Consumers of Healthcare. 
Timothy Bickmore, Daniel Mauer, Francisco Crespo and Thomas Brown. 2008. Negotiating Task Interruptions with Virtual Agents for Health Behavior Change. In Proceedings of the 7th International Joint Conference on Autonomous Agents and Multiagent Systems, 1241-1244.

Timothy Bickmore, Daniel Schulman and Candace Sidner. 2009. Issues in Designing Agents for Long Term Behavior Change. CHI'09 Workshop on Engagement by Design. 